\title{
Experimental observations of mill operation parameters on kinematic of the tumbling mill contents
}

\author{
M.M. Soleymani ${ }^{1, a}$, M. Fooladi Mahani ${ }^{2}$ And M. Rezaeizadeh ${ }^{3}$ \\ 1 Mechanical Engineering Department, Payame Noor University, Kerman, Iran \\ 2 Mechanical Engineering Department, Shahid Bahonar University, Kerman, Iran \\ 3 Mechanical Engineering Department, Graduate University of Advanced Technology, Kerman, Iran
}

Received 30 October 2014, Accepted 27 September 2015

\begin{abstract}
The present paper aims to investigate the key points of kinematic of contents inside the mill (load orientation), namely the shoulder, toe, impact, and pool angles. The kinematic of the mill contents in wet grinding is influenced by a range of parameters such as: media charge level (ball filling), slurry filling, and mill speed. In this work the influence of these operating parameters was investigated using a pilot mill $(1000 \times 500 \mathrm{~mm})$. To this end, a Copper ore $(-1000 \mu \mathrm{m})$ was used to prepare slurry at $50 \%$ solids concentration by mass. The tests covered a range of slurry filling $(U)$ from 0 to 2.92 with ball filling $24 \%$ and $30 \%$ of mill volume and 6 different speeds between $60 \%$ and $85 \%$ of critical speed. It is observed as the mill speed increases, the more the impact angle will decrease. Toe angle increases in the range 1525 degrees by formation of the pool in mill and exertion of the floating (buoyancy) forces to the particles inside the mill. The more the mill speed and the slurry filling increase, the more the shoulder angle will increase. The increase in slurry volume leads to the elevation of the pool level and therefore, the pool angle will exponentially decrease.
\end{abstract}

Key words: Mill / kinematic / shoulder angle / toe angle / wet grinding

\section{Nomenclature}

\begin{tabular}{|c|c|}
\hline $\bar{C}$ & Slurry concentration, mass fraction of solid (-) \\
\hline$d$ & Mill diameter $(\mathrm{m})$ \\
\hline$J_{B}$ & Fractional ball filling $(-)$ \\
\hline$J_{t}$ & Total charge filling $(-)$ \\
\hline$R$ & Mill radius $(\mathrm{m})$ \\
\hline$U$ & $\begin{array}{l}\text { Fraction of the interstices of the ball bed } \\
\text { filled by powder }(-)\end{array}$ \\
\hline$y$ & Height of the formed pool inside the mill (m) \\
\hline$\theta_{\text {impact }}$ & $\begin{array}{l}\text { Impact angle of the ball in cascading motion } \\
\text { (deg) }\end{array}$ \\
\hline$\theta_{\text {pool }}$ & $\begin{array}{l}\text { Pool angle of the free surface of the slurry } \\
\text { pool (deg) }\end{array}$ \\
\hline$\theta_{\text {shoulder }}$ & Shoulder of the medium charge angle (deg) \\
\hline$\theta_{\text {toe }}$ & Toe of the medium charge angle (deg) \\
\hline$\phi_{\mathrm{C}}$ & $\begin{array}{l}\text { Mill rotational speed as a fraction of the } \\
\text { criticalspeed }(-)\end{array}$ \\
\hline$\omega$ & Angular velocity ( $\mathrm{rad} / \mathrm{s})$ \\
\hline
\end{tabular}

\section{Introduction}

Grinding is one of the most important operational stages in the minerals processing. Ball mills and AG/SAG

\footnotetext{
a Corresponding author: mmsoleymani@pnu.ac.ir
}

mills are of the common types of milling. Around $30 \%$ of energy consumption in minerals processing goes to the milling process and only $5 \%-10 \%$ of the energy is consumed in the grinding process [1]. Most of the phenomena inside a mill are complicatedly dependent on geometrical parameters and physical properties. Studying the kinematic of the mill contents depends on the many parameters such as: mill speed, number, height and face-angle of lifters, size and volume of ball and ore, viscosity, and density and elevation of the slurry inside the mill $[2,3]$.

Tumbling mills include a rotary cylindrical pipe of 2.5$12 \mathrm{~m}$ diameter and a length of 3-5 m, made of steel plates and reaching up to $22 \mathrm{MW}$ consumption power. For the mill feed; the substance poured into the mill to be ground, can be either ore alone (AG mill) or ore with grinding medium (SAG mill). The grinding medium can be steel balls (Ball mill) or steel rod (Rod mill). Inside a mill, materials change from $5-250 \mathrm{~mm}$ dimensions into 10-300 mic due to impact and abrasion processes. For wear protection, the inner surface of the mill is covered by liner. Inside the mill, as shown in Figure 1, some steel profiles with a usually quadrilateral section (lifters) are applied in longitude direction so as to move the load inside the mill up to the specific height. The lifters under the rotary effect of the mill, move the feed up to the release point (Load shoulder). While the mill feed is coming up and due to 


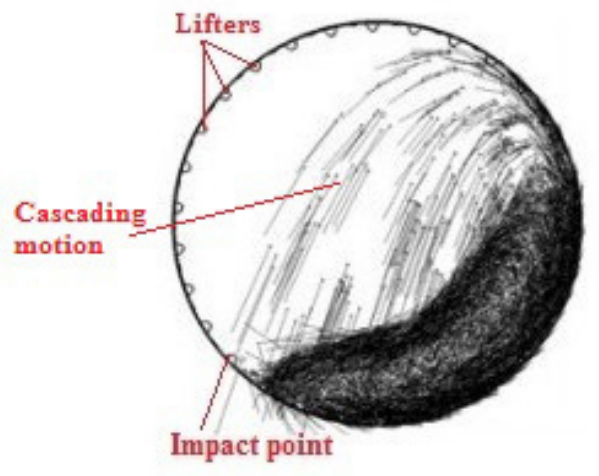

(a)

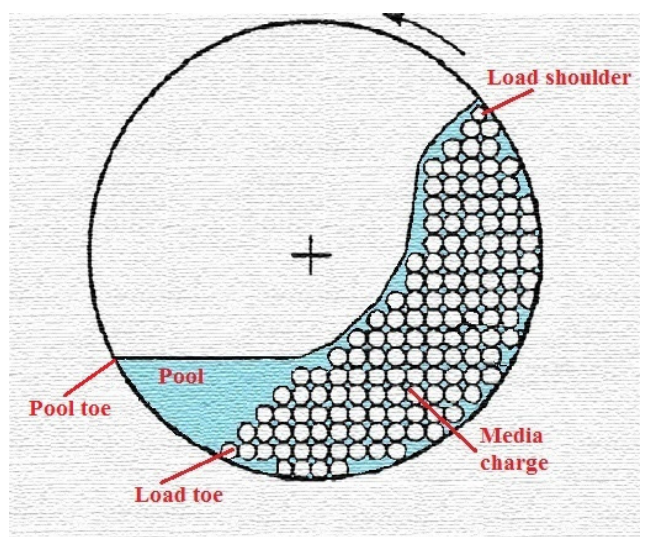

(b)

Fig. 1. Schematic of the load behavior in dry and wet tumbling mills. (a) SAG mill in dry condition. (b) Ball mill in wet condition.

their abrasion and erosion against each other, some of the grinding processes will be carried out. The release point is very crucial and depends on the height and face-angle of the lifters, mill speed, and friction coefficient between the feed and the lifters. Under the effect of their weight, the feed particles release from the shoulder point as a projectile and either during the path or at the end, will impact against each other and use the obtained kinetic energy from the previous stage, falling on the load toe for grinding (Impact point) $[1,2]$. Lifters fracture due to impact of balls to the lifters especially in variable-speed AG/SAG mill, is major issue. So knowing about the operation conditions and preventing impact balls and lifters, adjusting the impact point and the load toe is very crucial [4-6].

Maleki-Moghaddam, Yahyaei, and Banisi [7, 8] have developed an application in Microsoft Excel (called "GMT"), using Powell's analytic relations [9] with the aim to simulate the path for materials movement inside the mill. Moys et al. [10,11] have studied the dynamic behavior of the load in wet condition in industrial as well as pilot scale. Katubilwa and Moys [12] have conducted some experiments for different viscosities and $60 \%$ of the critical speed by means of a pilot mill $(552 \mathrm{~mm}$ diameter, $180 \mathrm{~mm}$ length, 18 lifters of $25 \mathrm{~mm}$ height and $20^{\circ}$ angle, 20\% charge of ball with $10 \mathrm{~mm}$ diameter); they made a fluid by combination of water and glycerin oil with different densities $1000-1202 \mathrm{~kg} . \mathrm{m}^{-3}$ (this density range is not applied in mineral industries) and then studied the effect of the slurry filling and viscosity inside the mill on the shoulder, toe, and pool angles. But in this article the real slurry (pulp) was used. Shi and NapierMunn [13] have studied the effect of slurry viscosity on grinding. Attia [14] and Nikravesh [15] using analytic relations for multi-body dynamics, and Cleary and Morrison $[16,17]$ have analyzed the experimental mill of $1.8 \mathrm{~m}$ diameter, $60 \mathrm{~cm}$ length in 3D mode by DEM-SPH method in wet conditions. Rezaeizadeh, et al. [18] have specified the speed of particles inside the mill in every point of the load orientation (i.e. from load toe to shoulder and from shoulder to re-fall on toe) by DEM and presented a pattern for wear by relative speed varieties. In order to easily model the behavior of the mill contents EbrahimiNejad and Fooladi divided the movement of a particle inside the mill into three steps and then studied each one separately [19].

In this work, the effect of the operating parameters of tumbling mill, slurry filling, ball filling, and mill speed on the load orientation was experimentally investigated using a large pilot mill. The present work provides further insight on the slurry pooling phenomenon and the novelty of this work is in investigating the impact angles.

\section{Experiments}

\subsection{Pilot mill}

The experiments were implemented by a $3 \mathrm{D}$ pilot mill of $100 \mathrm{~cm}$ diameter and $50 \mathrm{~cm}$ length. Front of mill is transparent and made of Perspex material. A section of the machine is displayed in Figure 2. There were 60 lifters of $14 \mathrm{~mm}$ height and 14.5-degree face angle. This pilot mill was capable of using different-size balls; however, just the $25 \mathrm{~mm}$ ones were used in this study as the solid load (ball filling); grinding media with $24 \%$, and $30 \%$ charges were applied. The mill motor was capable of changing the rotation permanently up to $100 \%$ of the critical speed. The critical speed for a grinding mill is defined as the rotational speed where centrifugal forces equal gravitational forces at the mill shell's inside surface. This is the rotational speed where balls will not fall away from the mill's shell. Critical speed is calculated from equation: $\omega_{c}=\sqrt{\frac{g}{R}}$. In this work, the mill radius is $0.5 \mathrm{~m}$ and $\omega_{\mathrm{c}}=4.43 \mathrm{rad} . \mathrm{s}^{-1}$ or $42 \mathrm{rpm}[1]$.

In every experimental condition, the mill was allowed to work for several rotations and then the videos and photos were taken to measure the key point of load. A high-speed camera (CASIO-EX-F1) was applied to take photos and films from the mill contents with $60-1200 \mathrm{fps}$. In order to prevent Perspex plate wetting by slurry, nano material with hydro-phobic behavior was sprayed on Perspex plate. The conditions of implementing the experiments are shown in Table 1. 

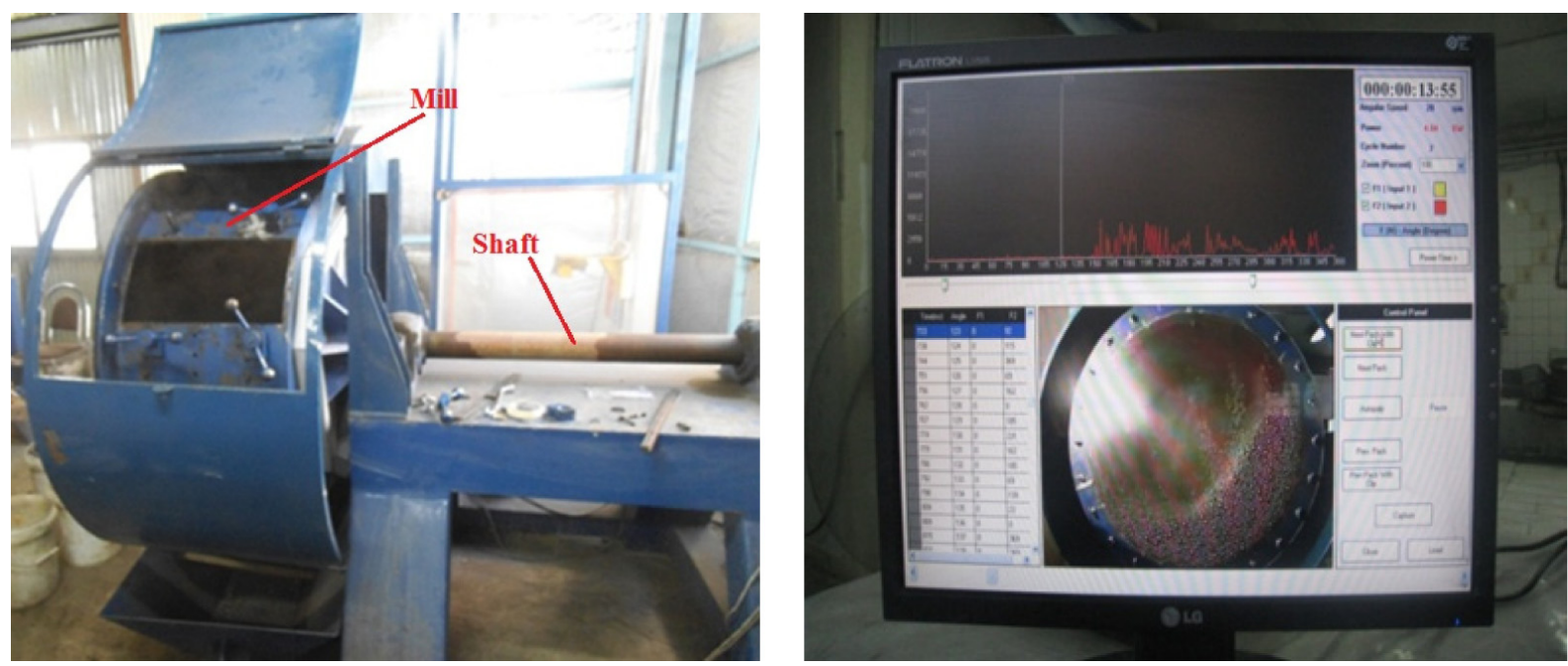

Fig. 2. View of the experimental pilot mill and the monitoring of the device.

Table 1. Conditions of implementing the experiments.

\begin{tabular}{|c|c|c|}
\hline \multirow{4}{*}{ Mill } & Diameter & $1000 \mathrm{~mm}$ \\
\hline & Length & $500 \mathrm{~mm}$ \\
\hline & Speeds & $25,27,29,31,33$ and $35 \mathrm{rpm}$ \\
\hline & Fraction of critical speed $\left(\Phi_{\mathrm{C}}\right)$ & $0.6,0.65,0.7,0.75,0.8$ and 0.85 \\
\hline \multirow{4}{*}{ Lifters } & Number & 60 \\
\hline & Shape & Trapezoid with $22 \mathrm{~mm}$ leg thickness \\
\hline & Height & $14 \mathrm{~mm}$ \\
\hline & Face angle & 14.5 degree \\
\hline \multirow{4}{*}{ Grinding media } & Material & Chrome alloy steel \\
\hline & Ball diameter & $25.4 \mathrm{~mm}(1 \mathrm{inch})$ \\
\hline & Density & $7800 \mathrm{~kg} \cdot \mathrm{m}^{-3}$ \\
\hline & Ball filling $\left(J_{\mathrm{b}}\right)$ & 0.24 and 0.30 fraction of mill volume \\
\hline \multirow{5}{*}{ Feed } & Material (size) & Copper ore $(-1000 \mu \mathrm{m})$ \\
\hline & Ore density & 2700 kg.m ${ }^{-3}$ \\
\hline & Slurry concentration $(C)$ & $50 \%$ (weight percent) \\
\hline & Slurry density & $1460 \mathrm{~kg} \cdot \mathrm{m}^{-3}$ \\
\hline & Slurry filling $(U)$ & $U=0-2.92$ (as volume fraction of ball bed voidage) \\
\hline
\end{tabular}

This experiment was partly based on taking videos and photos from the contents of a pilot mill; a scaled plate was placed on the fixed frame of the mill so as to observe the key-point angles of the load orientation after taking each photo. Two $500 \mathrm{~W}$ projectors were used for providing more light inside the mill for the photos to have a better quality. In each experiment, the load-orientation photos were taken within $5 \mathrm{~s}$ by a high-speed camera $(60 \mathrm{f} / \mathrm{s})$. From among 300 photos taken, key points of kinematic of contents inside the mill (load orientation) including the shoulder angle, toe angle, pool angle, and impact angle in wet conditions were extracted. Definition of the load behavior in mills is shown in Figure 1.

\subsection{Preparation of the feed samples}

The feed that was chosen for the pilot is a Sarcheshmeh copper ore in Iran that the specific density of the ore used as established in routine inspection is $2700 \mathrm{~kg} . \mathrm{m}^{-3}$. The supplied ore was a fine material of size distribution less than $1 \mathrm{~mm}$. In order to prepare samples of similar feed size distribution, conventional splitting methods were used. The mass of water was added to the powder sample in order to make up slurry of appropriate solids concentration. The fluid volume fraction in mill $(U)$ is the concept previously defined by Austin [20]. The tests covered slurry concentrations at $50 \%$ by weight and fractional interstitial bed filling $(U)$ from 0 to 2.5 , using batch grinding of a feed of -18 mesh $(1 \mathrm{~mm})$ copper ore. Slurry density was $1460 \mathrm{~kg} \cdot \mathrm{m}^{-3}$ (Table 1).

\subsection{Test method}

At first, the mill was filled by specific ball filling; then, it was tested by different speeds $(60-85 \%$ of the critical speed) in dry conditions. For each experimental condition, the mill was set to work for seconds to reach stability and then the videos and photos were taken. In the next step of the experiment, $5 \%$ of the mill volume was filled with feed and then the mill was tested at different speeds. More 
M.M. Soleymani et al.: Mechanics \& Industry 17, 408 (2016)

Table 2. Load orientation results in terms of slurry filling at different speeds for ball filling $24 \%$.

\begin{tabular}{|c|c|c|c|c|c|c|c|c|c|}
\hline$\% N_{\mathrm{C}}$ & Key points & $\bar{U}=0$ & $\bar{U}=0.41$ & $U=0.83$ & $U=1.25$ & $U=1.66$ & $U=2.08$ & $U=2.5$ & $U=2.92$ \\
\hline \multirow{4}{*}{$60 \%$} & $\theta_{\text {toe }}$ & 139 & 139 & 144 & 154 & 148 & 151 & 153 & 153 \\
\hline & $\theta_{\text {shoulder }}$ & 302 & 300 & 305 & 309 & 308 & 310 & 307 & 310 \\
\hline & $\theta_{\text {pool }}$ & - & - & - & 120 & 114 & 108 & 105 & 101 \\
\hline & $\theta_{\text {impact }}$ & 173 & 170 & 171 & 161 & 162 & 164 & 164 & 160 \\
\hline \multirow{4}{*}{$65 \%$} & $\theta_{\text {toe }}$ & 143 & 139 & 138 & 155 & 150 & 153 & 153 & 155 \\
\hline & $\theta_{\text {shoulder }}$ & 309 & 307 & 308 & 312 & 313 & 312 & 316 & 313 \\
\hline & $\theta_{\text {pool }}$ & - & - & - & 119 & 115 & 109 & 105 & 100 \\
\hline & $\theta_{\text {impact }}$ & 166 & 169 & 165 & 166 & 163 & 160 & 150 & 145 \\
\hline \multirow{4}{*}{$70 \%$} & $\theta_{\text {toe }}$ & 143 & 134 & 136 & 155 & 154 & 154 & 154 & 151 \\
\hline & $\theta_{\text {shoulder }}$ & 313 & 311 & 311 & 313 & 317 & 316 & 318 & 318 \\
\hline & $\theta_{\text {pool }}$ & - & - & - & 121 & 114 & 108 & 104 & 100 \\
\hline & $\theta_{\text {impact }}$ & 151 & 157 & 159 & 153 & 149 & 144 & 139 & 137 \\
\hline \multirow{4}{*}{$75 \%$} & $\theta_{\text {toe }}$ & 142 & 137 & 139 & 159 & 157 & 155 & 154 & 149 \\
\hline & $\theta_{\text {shoulder }}$ & 314 & 317 & 316 & 319 & 319 & 320 & 322 & 321 \\
\hline & $\theta_{\text {pool }}$ & - & - & - & 121 & 115 & 109 & 104 & 99 \\
\hline & $\theta_{\text {impact }}$ & 149 & 140 & 134 & 141 & 139 & 136 & 134 & 133 \\
\hline \multirow{4}{*}{$80 \%$} & $\theta_{\text {toe }}$ & 142 & 143 & 139 & 150 & 153 & 154 & 151 & 154 \\
\hline & $\theta_{\text {shoulder }}$ & 322 & 319 & 322 & 323 & 325 & 323 & 323 & 324 \\
\hline & $\theta_{\text {pool }}$ & - & - & - & 120 & 115 & 109 & 104 & 98 \\
\hline & $\theta_{\text {impact }}$ & 138 & 139 & 132 & 137 & 132 & 130 & 123 & 120 \\
\hline \multirow{4}{*}{$85 \%$} & $\theta_{\text {toe }}$ & 138 & 136 & 142 & 159 & 150 & 155 & 153 & 157 \\
\hline & $\theta_{\text {shoulder }}$ & 324 & 326 & 325 & 329 & 327 & 328 & 332 & 333 \\
\hline & $\theta_{\text {pool }}$ & - & - & - & 121 & 115 & 107 & 103 & 98 \\
\hline & $\theta_{\text {impact }}$ & 124 & 126 & 127 & 124 & 122 & 118 & 121 & 117 \\
\hline
\end{tabular}

feeds were added in each step and the tests were repeated at different speeds for the fluid volume inside the mill to reach more than twice as much as the ball filling. The slurry temperature was permanently measured during the tests, the changes of which being observed to be $30-34^{\circ} \mathrm{C}$.

\section{Results and discussion}

Summary of the load orientation results in terms of slurry filling at different speeds for ball filling $24 \%$ and $30 \%$ is presented in Tables 2 and 3 . To this end, a photographic technique was employed to observe the dynamics behavior of the mill contents with transparent pilot mill. The captured photos were uploaded on a computer, then analyzed frame after frame to get an accurate measurement of the pool position and the toe, shoulder and impact positions of the medium charge. A scaled plate was placed on the fixed frame of the mill, so for each and every frame retained for analysis, key angular positions of the charge were accurately measured with the 12 o'clock position being $0^{\circ}$ while the 9,6 and 3 o'clock position was used as $90^{\circ}, 180^{\circ}$ and $270^{\circ}$, respectively. Figure 3 illustrates how the toe and shoulder of medium charge, top impact point of balls and pool angle were determined frame by frame. Perspex plate was wetted by slurry and some of photos did not have good quality. A few photos that had good quality were selected to measure the angles and average angles of the key positions of the load were determined. Their variability is seen to be within $\pm 3^{\circ}$ at $90 \%$ confidence level.
According to Tables 2 and 3 , for $U$ less than 1 , no pool was formed inside the mill. The mill speed variety did not influence (negligible) the toe and pool angles. Also, the change in shoulder angle and the impact angle influenced by the level of the fluid inside the mill was insignificant. Figures 4 to 7 shows the changes of each key point of the load orientation for grinding media of $24 \%$ and $30 \%$.

The impact angle is presented in this article for the first time alongside other parameters during the slurry pool formation. The impact angle $\theta_{\text {impact }}$ mostly depends on the mill speed rather than on the $U$. According to Tables 2 and 3 and Figure 4, an increase of one percent at the critical speed of mill will lead to the impact angle decrease of two degrees. For example with the increase of mill speed from $60 \%$ to $65 \%$, the impact angle decreases 9 degrees $(174-165)$ at $U=0,10$ degrees $(176-166)$ at $U=1$, and 10 degrees (164-154) at $U=2$. With the increase in fluid and the subsequent increase in volume of the mill contents, the impact angle decreased very little and only in high speeds. As shown in Figure 4, the more the mill speed is increased, the more the shoulder angle and the release speed of the ball from the mill shell will increase; the balls, however, will pass shorter flying paths and the hitting angle will decrease. Consequently, the balls will directly hit the lifters and may damage them instead of landing on the load toe. Visual inspection and model sense-making have allowed us to identify a good model in the description of the relationship between impact angle with fraction of critical speed $\Phi_{\mathrm{c}}$ and slurry filling $U$. A power function was found to be a good candidate 
M.M. Soleymani et al.: Mechanics \& Industry 17, 408 (2016)

Table 3. Load orientation results in terms of slurry-filling at different speeds for ball filling $30 \%$.

\begin{tabular}{|c|c|c|c|c|c|c|c|c|c|}
\hline$\% N_{\mathrm{C}}$ & Key points & $U=0$ & $U=0.33$ & $U=0.66$ & $U=1$ & $U=1.33$ & $U=1.66$ & $U=2$ & $U=2.33$ \\
\hline \multirow{4}{*}{$60 \%$} & $\theta_{\text {toe }}$ & 130 & 133 & 130 & 131 & 146 & 141 & 140 & 143 \\
\hline & $\theta_{\text {shoulder }}$ & 313 & 309 & 314 & 310 & 312 & 312 & 313 & 310 \\
\hline & $\theta_{\text {pool }}$ & - & - & - & - & 120 & 109 & 104 & 100 \\
\hline & $\theta_{\text {impact }}$ & 174 & 173 & 172 & 176 & 169 & 165 & 164 & 157 \\
\hline \multirow{4}{*}{$65 \%$} & $\theta_{\text {toe }}$ & 129 & 131 & 127 & 130 & 138 & 142 & 141 & 143 \\
\hline & $\theta_{\text {shoulder }}$ & 315 & 315 & 319 & 311 & 313 & 319 & 315 & 314 \\
\hline & $\theta_{\text {pool }}$ & - & - & - & - & 118 & 110 & 105 & 101 \\
\hline & $\theta_{\text {impact }}$ & 165 & 168 & 169 & 166 & 159 & 155 & 154 & 147 \\
\hline \multirow{4}{*}{$70 \%$} & $\theta_{\text {toe }}$ & 128 & 129 & 126 & 132 & 141 & 139 & 144 & 144 \\
\hline & $\theta_{\text {shoulder }}$ & 319 & 316 & 324 & 318 & 319 & 323 & 318 & 317 \\
\hline & $\theta_{\text {pool }}$ & - & - & - & - & 119 & 110 & 105 & 102 \\
\hline & $\theta_{\text {impact }}$ & 162 & 160 & 158 & 150 & 155 & 150 & 149 & 143 \\
\hline \multirow{4}{*}{$75 \%$} & $\theta_{\text {toe }}$ & 126 & 128 & 125 & 129 & 139 & 144 & 140 & 146 \\
\hline & $\theta_{\text {shoulder }}$ & 327 & 321 & 328 & 330 & 324 & 324 & 326 & 329 \\
\hline & $\theta_{\text {pool }}$ & - & - & - & - & 119 & 110 & 104 & 100 \\
\hline & $\theta_{\text {impact }}$ & 142 & 147 & 140 & 146 & 142 & 138 & 136 & 140 \\
\hline \multirow{4}{*}{$80 \%$} & $\theta_{\text {toe }}$ & 122 & 130 & 126 & 127 & 139 & 144 & 141 & 147 \\
\hline & $\theta_{\text {shoulder }}$ & 332 & 328 & 329 & 331 & 333 & 326 & 331 & 331 \\
\hline & $\theta_{\text {pool }}$ & - & - & - & - & 119 & 110 & 106 & 100 \\
\hline & $\theta_{\text {impact }}$ & 127 & 131 & 130 & 130 & 132 & 123 & 121 & 122 \\
\hline \multirow{4}{*}{$85 \%$} & $\theta_{\text {toe }}$ & 119 & 128 & 124 & 128 & 140 & 140 & 145 & 146 \\
\hline & $\theta_{\text {shoulder }}$ & 340 & 338 & 335 & 334 & 337 & 332 & 338 & 344 \\
\hline & $\theta_{\text {pool }}$ & - & - & - & - & 120 & 109 & 106 & 100 \\
\hline & $\theta_{\text {impact }}$ & 122 & 126 & 125 & 122 & 126 & 119 & 117 & 119 \\
\hline
\end{tabular}

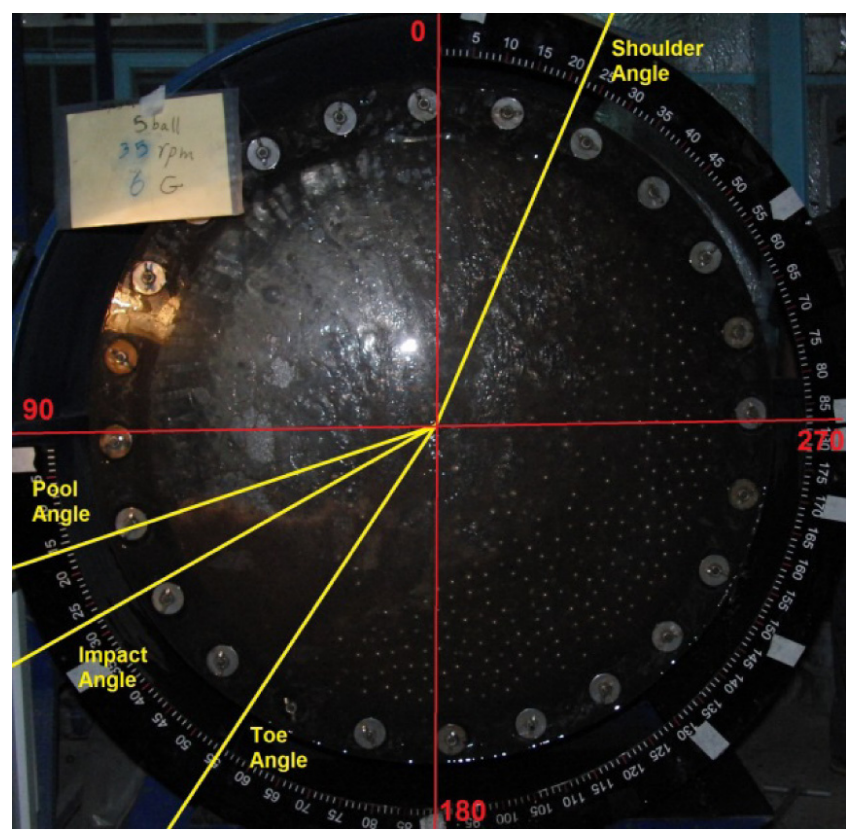

Fig. 3. Measurement of the key points of load orientation by photos.

for the description of our laboratory results. The model of the impact angle is presented below for ball filling 30\%:

$$
\theta_{\text {impact }}=93.1 \Phi_{\mathrm{c}}^{-1.656} \mathrm{e}^{-0.056 U}
$$

where $\Phi_{\mathrm{c}}$ is fraction of critical speed and $U$ is slurry filling.
According to Figure 5, the dependence of toe angle $\theta_{\text {toe }}$ on the mill speed was very little and at $U=0 \sim 1$ was almost fixed. At the time the slurry pool was formed inside the mill, $\theta_{\text {toe }}$ had a jump and increased up to $15 \sim$ 25 degrees. That was due to the increase in floatation (Archimedes or buoyancy) force because of the pool formation as well as the floatation of the balls; for $U>1.25$, the toe angle for $24 \%$ charge was almost fixed whereas for $U>1.33$, and $30 \%$ charge, it showed an increase. The movement of the toe was calculated by $\Delta s=R \Delta \theta_{\text {toe }}$, where $R$ is the mill radius. By the formation of pool in an industrial mill with $10 \mathrm{~m}$ diameter, the toe angle increased up to 0.35 radian (20 degrees); then the location of the toe can move $\Delta s=1.74 \mathrm{~m}$; that is a significant amount. In the discussion of the toe angle model, it is argued that the proposed power function responded very well to the experimental results. It offers discrepancies not exceeding $\pm 2^{\circ}$ at $95 \%$ confidence level. Last, in order to identify the trends of toe angle data in the pilot shown in Figure 5, the following function was used:

$$
\theta_{\text {toe }}=122.7 \Phi_{\mathrm{c}}^{-0.049} \mathrm{e}^{0.066 U}
$$

According to Figures 4 and 5, for charge of $24 \%$, prior to the slurry pool formation and for speeds of $80 \%$ and $85 \%$ of critical speed, the impact of balls point is higher than the toe one. This would cause a direct impact of balls with the wall of the mill which in turn would lead to lifters fracture. After the slurry pool formation and movement of the toe, in addition to the speeds of $80 \%$ and $85 \%$ of critical one, for speeds of $70 \%$ and $75 \%$ of critical one, the 


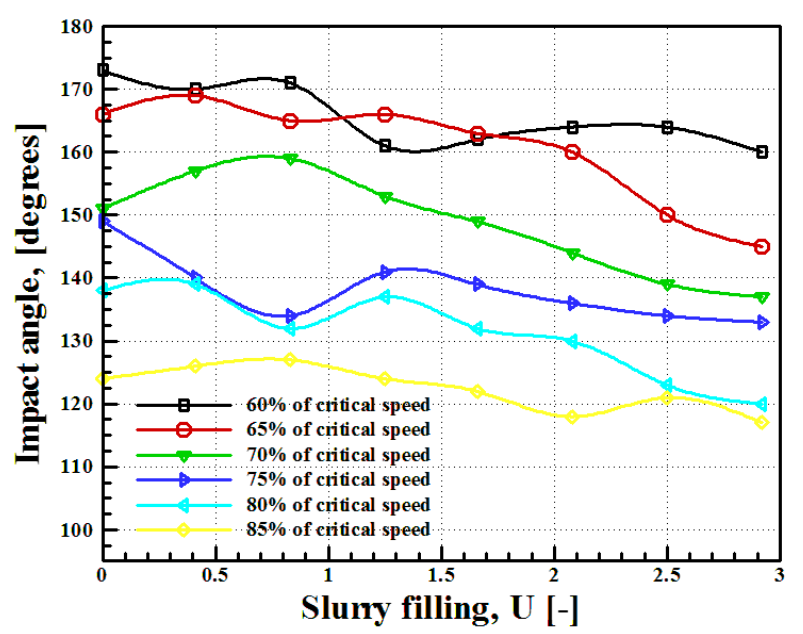

(a)

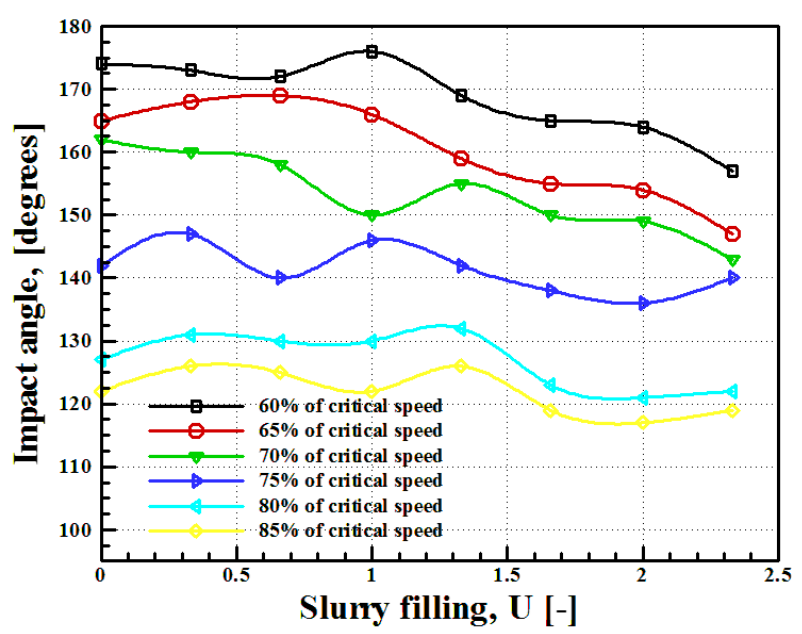

(b)

Fig. 4. Impact angle in terms of slurry-filling at different speeds. (a) Ball filling 24\%. (b) Ball filling $30 \%$.

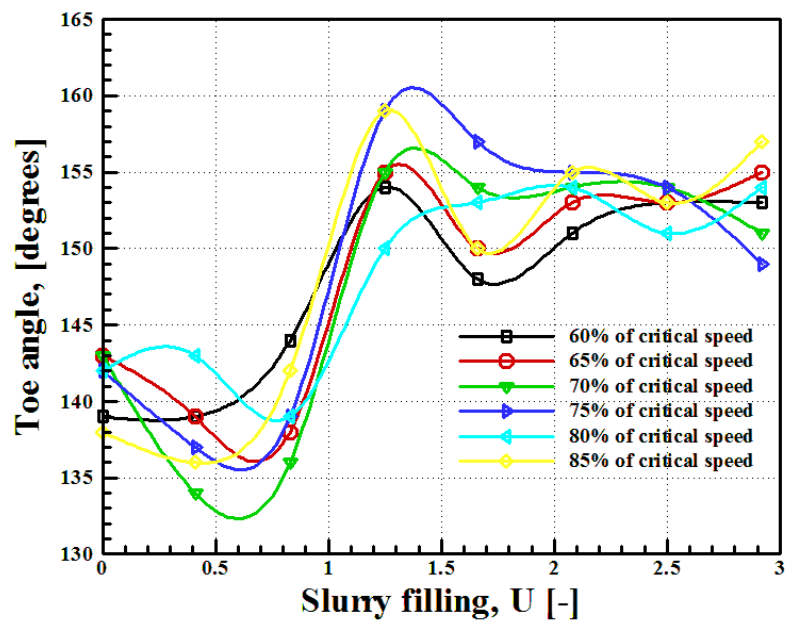

(a)

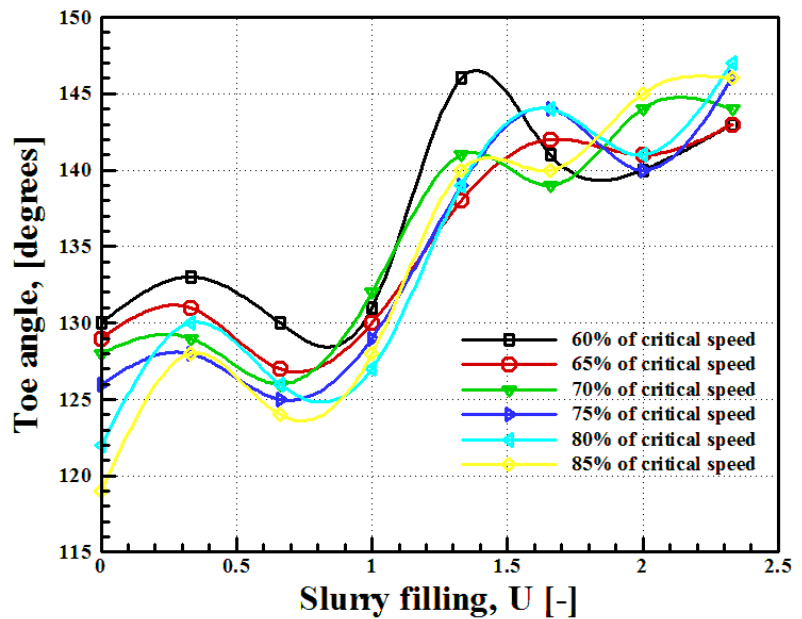

(b)

Fig. 5. Toe angle in terms of slurry-filling at different speeds. (a) Ball filling 24\%. (b) Ball filling $30 \%$.

impact of balls point is higher than the toe as well. For $30 \%$ of charge, due to the more charge, the direct impact of balls with the shell of the mill is less.

The shoulder angle $\theta_{\text {shoulder }}$ mostly depends on the mill speed rather than on $U$; the increase in the fluid inside the mill, increased the $\theta_{\text {shoulder }}$ a little. As shown in Figure 6 , the increase in the mill speed and in the subsequent raise in the centrifugation force caused the balls to release in higher angles from the lifters, thus increasing the shoulder angle. Variation of the shoulder angle with slurry filling is similar to the work conducted by Katubilwa and Moys [12] in a small pilot mill. Regarding the chart in Figure 6, the increase in the mill speed by $1 \%$ of the critical speed will lead to an increase in the shoulder angle by 1 degree. For instance, by increasing the mill speed from $65 \%$ to $77 \%$ of the critical speed, the shoulder angle will have an increase of 12 degrees. Mathematically, this statement would be written as follows:

$$
\theta_{\text {shoulder }}=347.9 \Phi_{\mathrm{c}}^{0.228} \mathrm{e}^{0.0005 U}
$$

The pool inside the mill starts forming from $U>1.25$. According to Figure 7, the pool angle $\theta_{\text {pool }}$ is not dependent on the mill speed. By increase of $U$, the fluid level inside the mill elevated and the $\theta_{\text {pool }}$ exponentially decreased. The height of the formed pool inside the mill was calculated by the following equation, where $R$ is the mill radius.

$$
y=R-R \sin \left(\theta_{\text {pool }}-90\right)=R\left(1-\sin \left(\theta_{\text {pool }}-90\right)\right)
$$

The pool angle was 120 degrees at the time of formation and therefore, the pool height came to $25 \mathrm{~cm}$ in pilot mill with $1 \mathrm{~m}$ diameter. The increase in slurry level causes the pool angle to reach 100 degrees and its height to reach $40 \mathrm{~cm}$. Thus, it can be concluded that in an industrial mill with $10 \mathrm{~m}$ diameter, the height of the formed pool is 2.5 to 4 meters regarding the slurry filling. A power function was found for the description of pool angle results.

$$
\theta_{\text {pool }}=147.9 \Phi_{\mathrm{c}}^{-0.009} \mathrm{e}^{-0.168 U}
$$




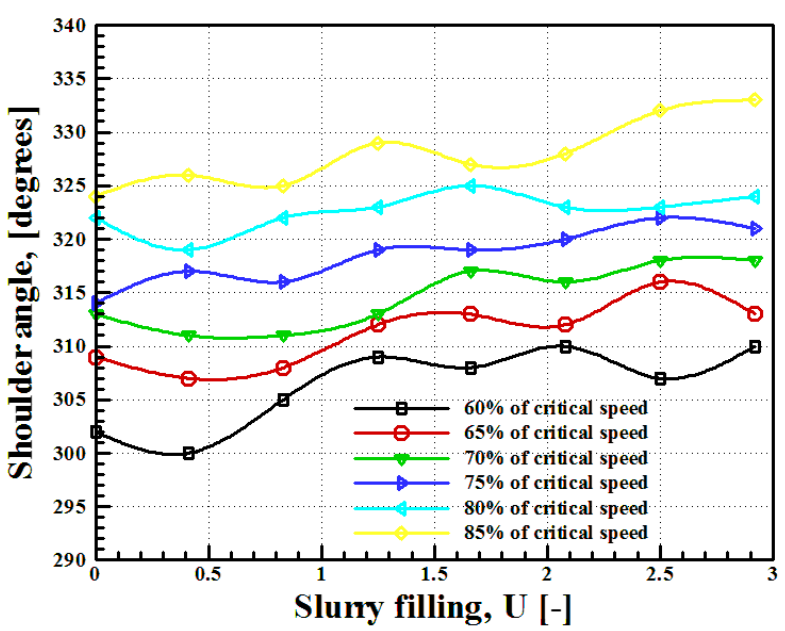

(a)

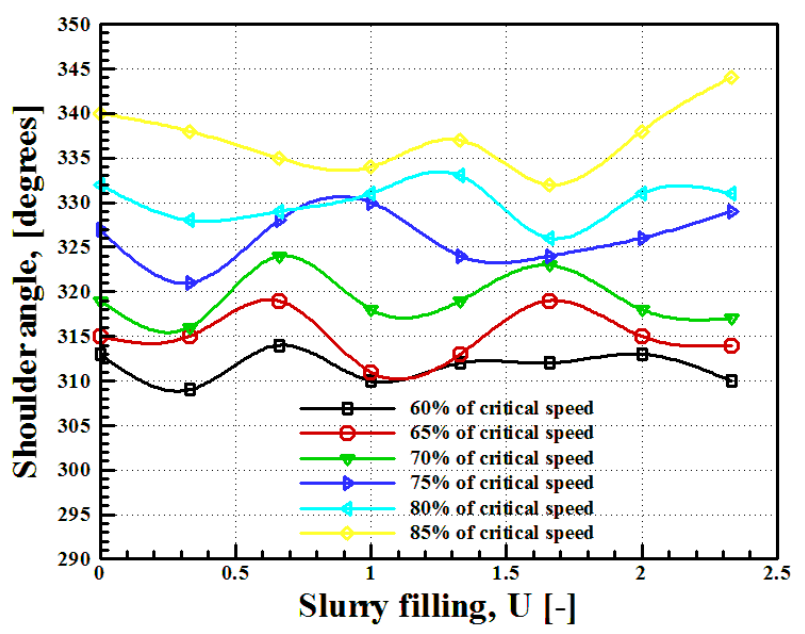

(b)

Fig. 6. Shoulder angle in terms of slurry-filling at different speeds. (a) Ball filling 24\%. (b) Ball filling $30 \%$.

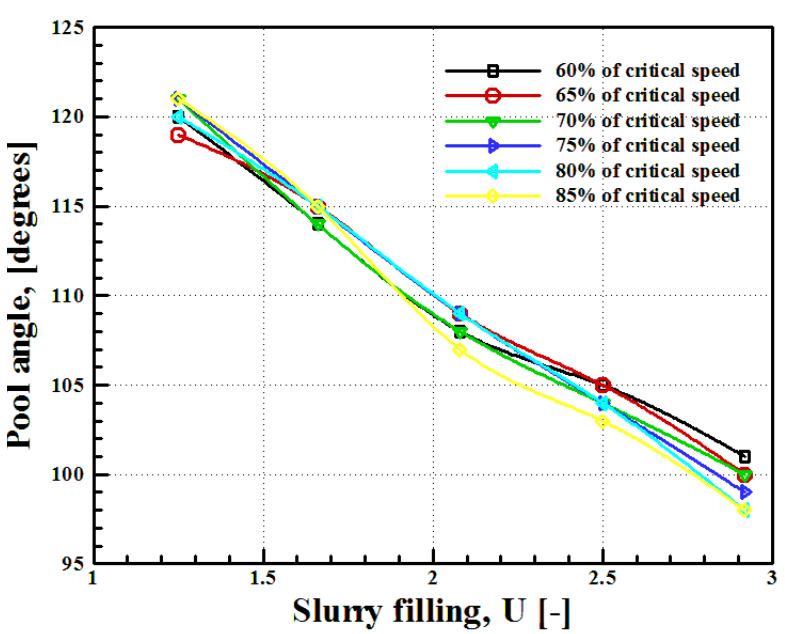

(a)

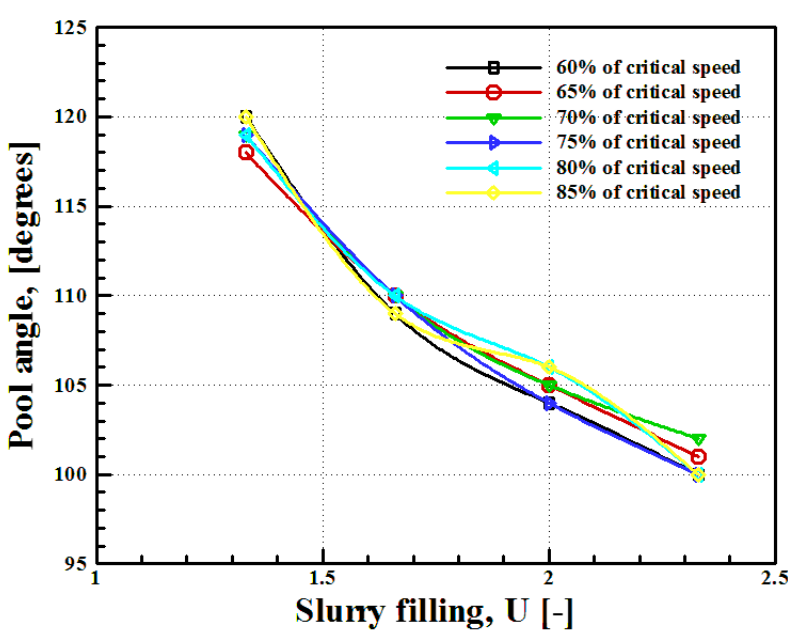

(b)

Fig. 7. Pool angle in terms of slurry-filling at different speeds. (a) Ball filling 24\%. (b) Ball filling $30 \%$.

Comparing Figure 4 with Figure 7 , all the balls fall into the slurry pool and their impact energy decreases. As a result, crushing will reduce.

\section{Conclusions}

The present paper aimed to study the effect of mill speed, ball filling, and slurry filling on the kinematic of the tumbling mill contents. The pilot mill $(1000 \times 500 \mathrm{~mm})$, initially loaded with balls at two different charges $24 \%$ and $30 \%$ volumetric filling, was run at six different speeds varying from $60 \%$ to $85 \%$ of the critical speed. The tests covered a range of slurry filling $(U)$ from 0 to 2.92 using of a feed of $-1 \mathrm{~mm}$ copper ore with $50 \%$ concentration. The following conclusions can be drawn from the present work:

- The impact angle decreases by the increase of the mill speed. An increase of $1 \%$ at the critical speed will lead to an impact angle decrease of 2 degrees. Also, with the increase of $U$, the decrease in impact angle will be very low and insignificant, being only observable for high speeds.

- The toe angle is not dependent on the mill speed. The toe angle jumps increasingly by formation of the pool inside the mill. The toe angle increased $15 \sim 25$ degrees by formation of the pool inside the mill and exertion of the buoyancy forces on the particles inside the mill. In wet condition, movement of the toe was calculated by $(0.13 \sim 0.22) d$, regarding the slurry filling and $d$ is the mill diameter.

- The shoulder angle increases by the increase in mill speed and fluid volume. An increase of $1 \%$ in the critical speed in mill speed will lead to a 1-degree increase in the shoulder angle.

- The pool angle is not dependent on the mill speed but will exponentially decrease by the increase in slurry volume. The height of the pool is $(0.25 \sim 0.4) d$, regarding the slurry filling. 
- The best result for variables condition of mill is $75 \%$ $80 \%$ of critical speed, more than $30 \%$ medium charge filling, and slurry filling before the pool formation $(0.80<U<1.25)$.

Acknowledgements. This research was accomplished by financial support of NICICO: special thanks to the generous helps of R\&D and the concentrate unit of NICICO; the authors would like to gratefully appreciate M. R. Yarahmadi and H. Mahmoodabadi for their help to this study.

\section{References}

[1] R.P. King, Modeling and simulation of mineral processing systems, Elsevier, 2001

[2] F.C. Bond, Crushing and grinding calculations, AllisChalmers Manufacturing Company, 1961

[3] B.K. Mishra, R.K. Rajamani, Simulation of charge motion in ball mills. Part 1: experimental verifications, Int. J. Mineral Process. 40 (1994) 171-186

[4] R. Rajamani, B. Mishra, Dynamics of ball and rock charge in sag mills. in Proc. SAG, 1996

[5] K.K. Kiangi, M.H. Moys, Measurement of the load behaviour in a dry pilot mill using an inductive proximity probe, Minerals Eng. 19 (2006) 1348-1356

[6] K.K. Kiangi, M.H. Moys, Particle filling and size effects on the ball load behaviour and power in a dry pilot mill: Experimental study, Powder Technol. 187 (2008) 79-87

[7] M. Maleki-Moghaddam, M. Yahyaei, S. Banisi, Converting AG to SAG mills: The Gol-E-Gohar Iron Ore Company case, Powder Technol. 217 (2012) 100-106

[8] M. Yahyaei, S. Banisi, Spreadsheet-based modeling of liner wear impact on charge motion in tumbling mills, Minerals Eng. 23 (2010) 1213-1219
[9] M.S. Powell, The effect of liner design on the motion of the outer grinding elements in a rotary mill, Int. J. Mineral Process. 31 (1991) 163-193.

[10] M. Moys, The Dynamic Behaviour of the Load in a Wet High-speed Grinding Mill, in South African Institute of Mining and Metallurgy, Colloquium on "Milling", Randburg, 1987

[11] M.H. Moys, M.A. Van Nierop, I. Smit, Progress in measuring and modelling load behaviour in pilot and industrial mills, Minerals Eng. 9 (1996) 1201-1214

[12] F.M. Katubilwa, M.H. Moys, Effects of filling degree and viscosity of slurry on mill load orientation, Minerals Eng. 24 (2011) 1502-1512

[13] F.N. Shi, T.J. Napier-Munn, Effects of slurry rheology on industrial grinding performance, Int. J. Mineral Process. 65 (2002) 125-140

[14] H. Attia, Equations of motion of planar mechanical systems based on particle dynamics and a recursive algorithm, Proc. Instit. Mech. Eng. K 218 (2004) 31-38

[15] P. Nikravesh, Newtonian-based methodologies in multibody dynamics, Proce. Instit. Mecha. Eng. K 222 (2008) 277-288.

[16] P. Cleary, R. Morrison, Predicting patterns of slurry flow within and discharge from a $3 \mathrm{D}$ pilot SAG mill, in Proc. SAG, 2011

[17] P.W. Cleary, R.D. Morrison, Prediction of 3D slurry flow within the grinding chamber and discharge from a pilot scale SAG mill, Minerals Eng. 39 (2012) 184-195

[18] M. Rezaeizadeh, et al., A new predictive model of lifter bar wear in mills, Minerals Eng. 23 (2010) 1174-1181

[19] S. Ebrahimi-Nejad, M. Fooladi-Mahani, Optimizing the Characteristics of the Motion of Steel Balls and their Impact on Shell Liners in SAG Mills, Iranian J. Mech. Eng. 10 (2009) 6

[20] L.G. Austin, R.R. Klimpel, P.T. Luckie, Process engineering of size reduction: ball milling, Society of Mining Engineers of the American Institute of Mining, Metallurgical and Petroleum Engineers, 1984 